\title{
pH-sensitivity of YFP provides an intracellular indicator of programmed cell death
}

\author{
Bennett Young ${ }^{1}$, Raymond Wightman ${ }^{1}$, Robert Blanvillain², Sydney B Purcel', Patrick Gallois ${ }^{1 *}$
}

\begin{abstract}
Background: Programmed cell death (PCD) is an essential process for the life cycle of all multicellular organisms. In higher plants however, relatively little is known about the cascade of genes and signalling molecules responsible for the initiation and execution of PCD. To aid with the discovery and analysis of plant PCD regulators, we have designed a novel cell death assay based on low cytosolic pH as a marker of PCD.

Results: The acidification that occurs in the cytosol during plant PCD was monitored by way of the extinction of YFP fluorescence at low $\mathrm{pH}$. This fluorescence was recovered experimentally when bringing the intracellular pH back to 7, demonstrating that there was no protein degradation of YFP. Because it uses YFP, the assay is nonedestructive, does not interfere with the PCD process and allows time-lapse studies to be carried out. In addition, changes of sub-cellular localisation can be visualised during PCD using the protein of interest fused to RFP.

Coupled to a transient expression system, this $\mathrm{pH}$-based assay can be used to functionally analyse genes involved in PCD, using point mutations or co-expressing PCD regulators. Transfecting $m B A X$ and AtBl-1 in onion epidermal cells showed that the $\mathrm{pH}$ shift is downstream of PCD suppression by AtBI-1. In addition, this method can be used to score PCD in tissues of stably transformed transgenic lines. As proof of principle, we show the example of YFP extinction during xylogenesis in Arabidopsis. This demonstrates that the assay is applicable to PCD studies in a variety of tissues.

Conclusions: The observation that YFP fluorescence is lost during the plant PCD process provides a new tool to study the genetic regulation and cell biology of the process. In addition, plant cell biologists should make a note of this effect of PCD on YFP fluorescence to avoid misinterpretation of their data and to select a pH insensitive reporter if appropriate. This method represents an efficient and streamlined tool expected to bring insights on the process leading to the $\mathrm{pH}$ shift occurring during PCD.
\end{abstract}

\section{Background}

PCD is a universal process across multicellular organisms that is highly regulated and tightly controlled by many genes. These genes are expected to act together to form organised cascades culminating in cell death. There are a few assays, which can be used to monitor PCD in plants in order to analyse the function and interaction of specific genes. These assays score PCD at various steps in the PCD process and each has its limitations. Some are destructive such as TUNEL assays for detecting the DNA fragmentation induced during PCD [1]. This assay involves fixing and permeabilising cells

\footnotetext{
* Correspondence: patrick.gallois@manchester.ac.uk

'Faculty of Life Sciences, Michael Smith Building, University of Manchester, Oxford Road, Manchester M13 9PT, UK

Full list of author information is available at the end of the article
}

before labelling DNA fragment 3' ends using a terminal deoxynucleotidyl transferase and labelled dUTP (fluorescein, biotin, digoxigenin). Enzymatic assays for caspaselike proteases have become relatively prevalent in the plant literature as many synthetic caspase substrates are now commercially available [2]. Most chromogenic or fluorogenic substrates are based on a four amino acid peptide with has a higher affinity for a subset of animal caspases e.g. DEVD for caspase3 and 7. Typically plant protein extracts are buffered at $\mathrm{pH} 7$ or 5.5 and incubated with one of the substrates at 50 to $100 \mu \mathrm{M}$. In addition, none destructive in situ caspase assay can be carried out using permeable caspase inhibitors coupled to the fluorescent molecule carboxyfluorescein [3]. The reagent easily permeates the cells and can irreversibly bind to caspase-like proteases inside the cell. Unbound

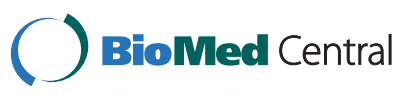


inhibitor molecules are washed away to eliminate background. In addition, two types of cell permeable substrates have been used in pollen for in vivo studies of caspase activation [4]. Biotum ${ }^{\mathrm{TD}}$ has developed a cell permeable substrate (Nucview 488) which when cleaved by caspase-like proteases releases a DNA dye which migrates to the cell nucleus and stains nuclear DNA [4]. The other substrate, CR(DEVD) $)_{2}$, is composed of two DEVD peptides coupled to the fluorophore cresyl violet (CR). Upon cleavage, the fluorescent CR marker is released as a red fluorescent product [4]. Recently, Zhang et al. 2009 [5] developed an in vivo PCD assay based on expressing a recombinant protein in cells that is a DEVD_FRET substrate cleaved by caspase3-like proteases. In this case, the fluorescence is lost when the substrate is cleaved. It requires costly confocal equipment. Finally, the dye mitotracker red can be used to detect a loss of mitochondrial membrane potential ( $\psi$ mit) in cells undergoing PCD. A loss of $\psi$ mit has been reported e.g. in tobacco cells during heat-shock induced PCD [6] and during tracheary element formation [7]. This dye is a cationic lipophilic fluorochrome, which acts by accumulating in the negatively charged matrix of the mitochondria. The accumulation of this probe in the mitochondria is dependent upon the strength of the $\psi$ mit, the loss of which results in a proportional loss of mitotracker fluorescence [8].

Other generic live/dead assays used in PCD studies are non-destructive such as when using fluorescein diacetate (FDA) in an in vivo enzymatic assay. The assay relies on cellular esterase activity as a marker of live cells; the enzyme converts the non-fluorescent FDA to fluorescein [9]. Because FDA is used to score live cells, a time course of life to death cannot be generated in the same cell as FDA fluorescence persists in dead cells. Further assays are based on the loss of membrane permeability that occurs during PCD. The molecules Evans blue, sytox green and sytox orange are excluded from live cells and only diffuse into the cells when membrane permeability is compromised. Evans blue labels the whole cell blue while sytox green and sytox orange light up the nucleus after binding to DNA $[10,11]$.

As plant vacuoles are acidic with a $\mathrm{pH}<6$ [12], it is logical to expect that vacuole rupture during PCD could result in cytosolic acidification. For example, authors of xylem differentiation studies had suggested that a cytosolic $\mathrm{pH}$ drop may occur when the vacuole ruptured during xylem PCD [13,14]. In addition, vacuole disruption was proposed as a defining characteristic for bona fide PCD as vacuole collapse was reported not to occur in necrotic cell death [15]. During PCD activated by self-incompatibility (SI) in the pollen tubes of Papaver rhoeas, Bosch et al. 2007 [4] were able to measure a dramatic drop of intracellular $\mathrm{pH}$. Using a $\mathrm{pH}$ sensitive probe, they found that the intracellular $\mathrm{pH}$ of pollen tubes undergoing SI dropped from $\mathrm{pH} 7$ to $\mathrm{pH} 5.5$. A pH of 5.5 is remarkably close to the $\mathrm{pH}$ optima measured in vitro for most of the caspase-like activities detected during plant PCD [16], suggesting that lowering the $\mathrm{pH}$ may be part of the activation process for caspase-like activities in plants.

Evidence in the literature $[4,14]$ and our own observation that YFP fluorescence was lost during the PCD process led us to consider that a large $\mathrm{pH}$ drop was a general feature of plant PCD and therefore a good candidate to develop a novel marker for PCD in plant cells.

\section{Results}

BAX induces PCD and intracellular acidification in onion cells

Transfection of onion epidermal cells using biolistics is a common technique used in many laboratories to examine the sub cellular localisation of proteins or to confirm protein-protein interactions using split YFP approaches [17-19]. Onion cells constitute an excellent experimental model, showing little background fluorescence compared to green tissues. To induce PCD and measure $\mathrm{pH}$ change in onion cells, we selected the mammalian proapoptotic gene $B A X$. There is no known homologue of $B A X$ in plants but despite this, $B A X$ induced hallmarks of PCD in tobacco plants [20] and in Arabidopsis $[21,22]$. BAX::YFP under the control of the $35 \mathrm{~S}$ promoter was bombarded into onion cells. $B A X:: Y F P$ has been shown to be targeted to mitochondria [22] and consistent with this, YFP fluorescence took a punctuate appearance in onion cells (Figure 1A). To ensure that BAX was capable of inducing bona-fide PCD in our system, induction of caspase-like protease activity, plasma membrane retraction and loss of mitochondrial membrane potential were investigated. At 30 hours, control cells treated with mitotracker showed a labelling distinctive of mitochondria while $B A X$ expressing cells were all mitotracker negative (Figure 1A). Mitotracker is only labelling mitochondria that are functional and have a membrane potential ( $\psi$ mit). A loss of $\psi$ mit is an early event in BAX and Nitric Oxide (NO) induced PCD in plants; it occurs as a result of the formation of the Permeability Transition Pore (PTP) $[22,23]$. In a separate experiment, 35S:: BAX and 35S::td-tomato were co:bombarded in onion cells. At 48 hours, caspase-like activity was detected in situ in 53\% of transfected cells using a cell permeable, fluorescent pan-caspase inhibitor (FAMVAD-FMK (Figure 1B). Induction of caspase-like activity is considered a strong hallmark for plant PCD [2]. In addition, $51 \%$ of transfected cell displayed a cell collapse with retraction of the plasma membrane from the cell wall and an increase in the prominence of the nucleus (Figure 1B). Such a cellular collapse, termed PCD 


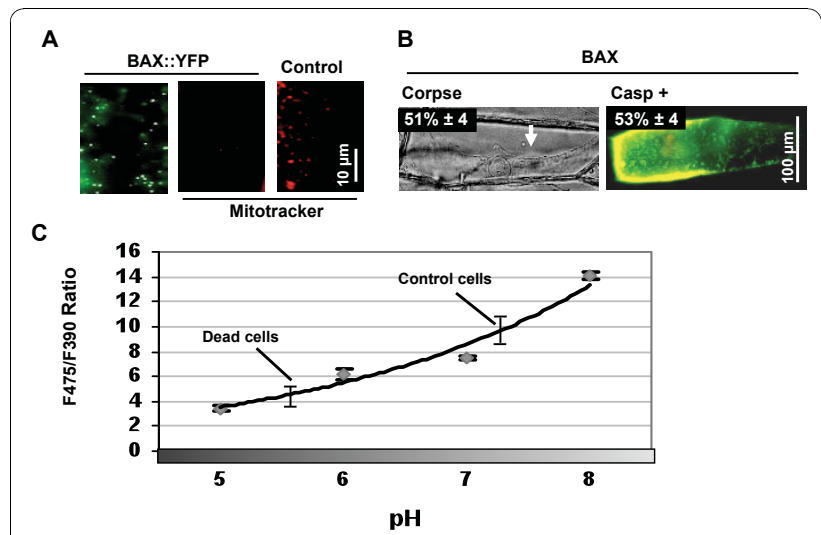

Figure $1 B A X$ expression induces $P C D$ and intracellular acidification. (A) Left panel; a close up of a p35S.:: BAX::YFP expressing cell showing typical punctuated subcellular localisation. Centre panel; all BAX::YFP expressing cells were negative for mitotracker ( $n=30)$. Right panel; untransfected cells were positive for mitotracker. Cells were observed $30 \mathrm{~h}$ post-bombardment. Scale bar $10 \mu \mathrm{m}$. (B) Corpse morphology (cellular collapse) and fluorescent VADase activity in cells expressing BAX $48 \mathrm{~h}$ postbombardment. White arrow shows plasma membrane retraction. For VADase, unlabelled p355::BAX was co-transfected with p35S::RFP$t d$-tomato in order to prevent overlap between the fluorescence of YFP and FAM-VAD-FMK. Inset panels show the percentage of BAX cells positive for these two PCD-hallmarks (values shown are minus the background obtained with an RFP-td-tomato control). Scale bar $100 \mu \mathrm{m}$. (C) Calibration curve for ptGFP readings in vivo. Cells were transfected with p35S ptGFP and incubated for 30 hours in the dark. Epidermal pieces were then incubated for 1 hour in $3 \mathrm{~mL}$ of buffer solution at various pHs with $0.003 \%$ Triton-20-X-100. F475/F390 ratiometric readings were then taken using 30 transfected cells for each $\mathrm{pH}$ value. Using the standard curve, the $\mathrm{pH}$ of control cells expressing ptGFP or of cells expressing BAX/ptGFP was interpolated using readings at $30 \mathrm{~h}$. The average for all control cells or for all low $\mathrm{pH}$ BAX cells was plotted on the standard curve. Error bars are for 51 control cells and 15 low pH cells.

corpse, has been shown to be specific to PCD as it is absent from plant cells that have died in an unregulated manner by necrosis [24]. The detection of these three PCD hallmarks confirmed that BAX induced PCD in onion cells.

To investigate the intracellular $\mathrm{pH}$ during $\mathrm{PCD}$ of onion cells, Ptilosarcus GFP (PtGFP) was chosen as a $\mathrm{pH}$ probe because of a broader $\mathrm{pH}$-responsiveness and a greater acidic-stability than other $\mathrm{pH}$ probes such as pHluorins [25]. First, onion epidermal cells were bombarded with $p t G F P$ under the $35 \mathrm{~S}$ promoter, permeabilised and equilibrated using buffers at $\mathrm{pH}$ ranging from 5 to 8. Fluorescent ratiometric measurements (F475/ F390) were taken. The calibration curve obtained (Figure 1C) fitted the first part of the sigmoidal curve described over a wider range of $\mathrm{pH}$ using recombinant ptGFP [25]. Next, 35S::ptGFP and 35S::BAX were cobombarded to induce PCD and measure intracellular $\mathrm{pH}$. After 30 hours of expression, all control cells expressing ptGFP only had an average intracellular $\mathrm{pH}$ of 7.3, a value typical for plant cell cytoplasm. By contrast at the same time point, cells co-expressing BAX and ptGFP had an acidic pH of 5.7 (Figure 1C). This $\mathrm{pH}$ value is consistent with the value of 5.5 reported by Bosch et al. 2007 [4] during PCD in pollen and is in support of using a buffer at $\mathrm{pH} 5.5$ to develop a marker of the PCD process in plant cells.

\section{$\mathrm{pH} 5.5$ attenuates the fluorescent signal of YFP in vivo}

It is known that YFP fluorescence can be reversibly attenuated at pH below 6, while RFP fluorescence is not affected [26-28]. To test if YFP could be used as an on/ off $\mathrm{pH}$ indicator for scoring low $\mathrm{pH}$ during $\mathrm{PCD}$ in vivo, onion epidermal cells were transfected with a $35 S:: Y F P$ construct, permeabilised and submitted to various $\mathrm{pH}$ treatments (Figure 2A). The buffering treatment used is the same as the one used to establish the $\mathrm{pH}$ calibration

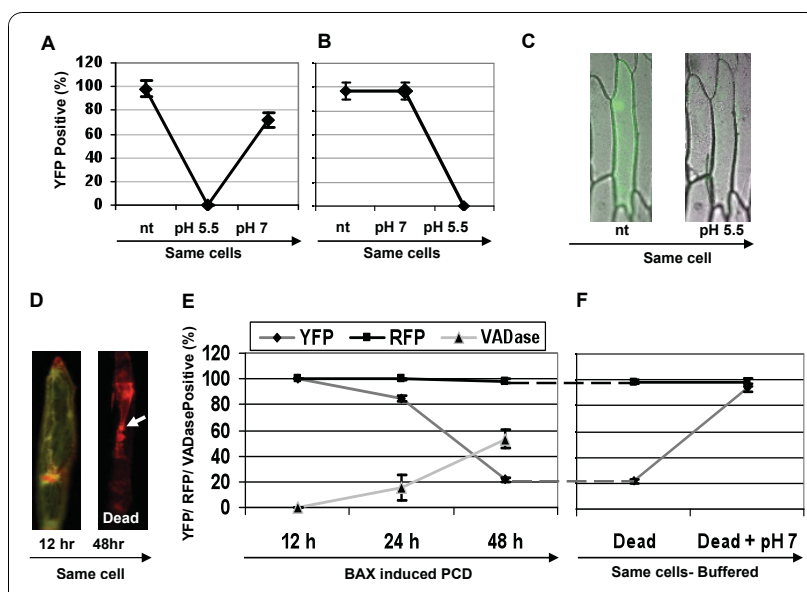

Figure 2 The attenuation during PCD of YFP fluorescence is due to low intracellular pH. (A, B) Epidermal cells were bombarded with p355::YFP and incubated in the dark for 24 hours. YFP fluorescence was scored three times in the same onion epidermal cells, first untreated (nt) set as 100\%, next buffered either at $\mathrm{pH} 5.5$ or $\mathrm{pH} 7$ and finally transferred to either $\mathrm{pH} 5.5$ or $\mathrm{pH} 7$. Modified intracellular $\mathrm{pH}$ was obtained by permeabilisation as in figure $1 C$. (C) Fluorescence images of one representative epidermal cell expressing $p 355::$ YFP for 24 hours, first untreated (nt) and next buffered to $\mathrm{pH} 5.5$; bright field and green channel have been merged. (D) Red and green merged images of the same cell coexpressing BAX::YFP and RFP-td-tomato. Before PCD (12 hr postbombardment) both YFP and RFP fluorescence are detectable. After PCD (48 hr) only RFP fluorescence is detectable. White arrow shows plasma membrane retraction characteristic of PCD cell collapse. (E) BAX-induced PCD: the same cells co-expressing BAX::YFP and RFPtd-tomato were scored for YFP and RFP fluorescence at 12, 24 and 48 hours post-bombardment. Incubation with FAM-VAD-FMK allowed caspase-like protease activity to be detected in cells transfected with p35S.:BAX (values shown are minus the background obtained with an RFP-td-tomato control). (F) At 48 hours, the same cells were buffered to $\mathrm{pH} 7$ (as in A-B) and the YFP and RFP fluorescence was re-scored. Errors bars represent $2 \times$ SE of triplicates of 100 cells each. 
curve in Figure 1. Buffers at $\mathrm{pH} 7$ and $\mathrm{pH} 5.5$ were chosen in order to mimic the $\mathrm{pH}$ drop measured in onion cells expressing BAX. The number of fluorescent cells in each sample was counted three consecutive times: 1) untreated, 2) after a first $\mathrm{pH}$ treatment and 3) after a second $\mathrm{pH}$ treatment (Figure 2A, B and 2C). A drop to $\mathrm{pH} 5.5$ completely extinguished the fluorescence in expressing cells (Figure 2C) and when the same cells were subsequently buffered back to $\mathrm{pH} 7$, a majority of cells recovered the YFP signal (Figure 2A). The pH 5.5 treatments therefore only attenuated the signal of YFP and did not permanently denature the protein. Since $\mathrm{pH}$ 7 as a first treatment had no effect on fluorescence (Figure $2 \mathrm{~B}$ ), it can be deduced that it is the $\mathrm{pH}$ and not the molarity of the buffer applied that had an effect on the fluorescence of YFP. By contrast and as expected, RFP was resistant to intracellular $\mathrm{pH}$ changes, as the $\mathrm{pH} 5.5$ treatment had no effect on the number of RFP positive cells (data not shown). The above reconstruction experiments using buffers suggested that an intracellular $\mathrm{pH}$ drop from 7 to 5.5 in the cytosol should attenuate the fluorescence of YFP to the point that it became undetectable. This was not the case for the fluorescence of RFP, which could be used as a reference gene for transfection.

\section{PCD attenuates YFP but not RFP fluorescence in vivo}

In order to test whether YFP fluorescence attenuation by low $\mathrm{pH}$ could be used to score PCD in vivo, we co-transfected cells with RFP td-tomato and $B A X$ fused to YFP $(B A X:: Y F P)$ (Figure 2D, E). The number of YFP and RFP expressing cells were counted at $12 \mathrm{~h}, 24 \mathrm{~h}$ and $48 \mathrm{~h}$ after bombardment (Figure 2E). At $12 \mathrm{~h}$, every transfected cell was expressing both constructs and the number of BAX::YFP and RFP fluorescent cells was the same. At $24 \mathrm{~h}$ post-bombardment, the cells were re-counted and there was a small reduction $(<20 \%)$ in the number of cells fluorescent for both BAX::YFP and RFP. By the $48 \mathrm{~h}$ time point, there was an $80 \%$ reduction in the number of BAX::YFP fluorescent cells. For RFP, the number of fluorescent cells remained unchanged across all time points. To investigate the correlation between loss of YFP fluorescence and PCD, caspase-like protease activity was followed in cells expressing $B A X$. For this purpose, cells transfected with $355: \because B A X$ were incubated with FAM-VAD-FMK. The number of cells exhibiting caspase-like protease activity across the time points was found to correlate with the number of cells with an attenuated YFP signal (Figure 2E).

To confirm that the attenuation of YFP fluorescence observed in cells expressing BAX::YFP and RFP tdtomato (Figure 2D) was due to a $\mathrm{pH}$ drop and not to protein degradation, we buffered these cells to $\mathrm{pH} 7$ for 1 hour. The lost BAX::YFP fluorescence was recovered in $95 \%$ of cells (Figure 2F). This confirmed that the $\mathrm{pH}$ drop measured during PCD is responsible for the loss of the YFP signal, while having no effect on RFP fluorescence.

\section{YFP can form the basis of a pH cell death assay to study} the interaction of genes regulating PCD

Having shown that YFP can be used as a PCD marker, we set up a transient assay to study PCD genetic regulation. To illustrate the principle (Figure 3), a plasmid expressing a $B A X-Y F P$ fusion was bombarded in onion cells either on its own or co-bombarded with a twomolar excess of plasmid expressing a PCD suppressor. The suppressor genes used were previously shown to suppress PCD in plants: the human anti-apoptotic gene Bcl-2 [29] and the anti-PCD Arabidopsis BAX-I (AtBI-1) $[21,22]$. Finally, a GUS expressing plasmid was included in all bombardments to use GUS activity as a pH insensitive reference. Note that GUS histochemical assays are carried out using a buffered solution and therefore scoring GUS expression is not affected by the $\mathrm{pH}$ shift

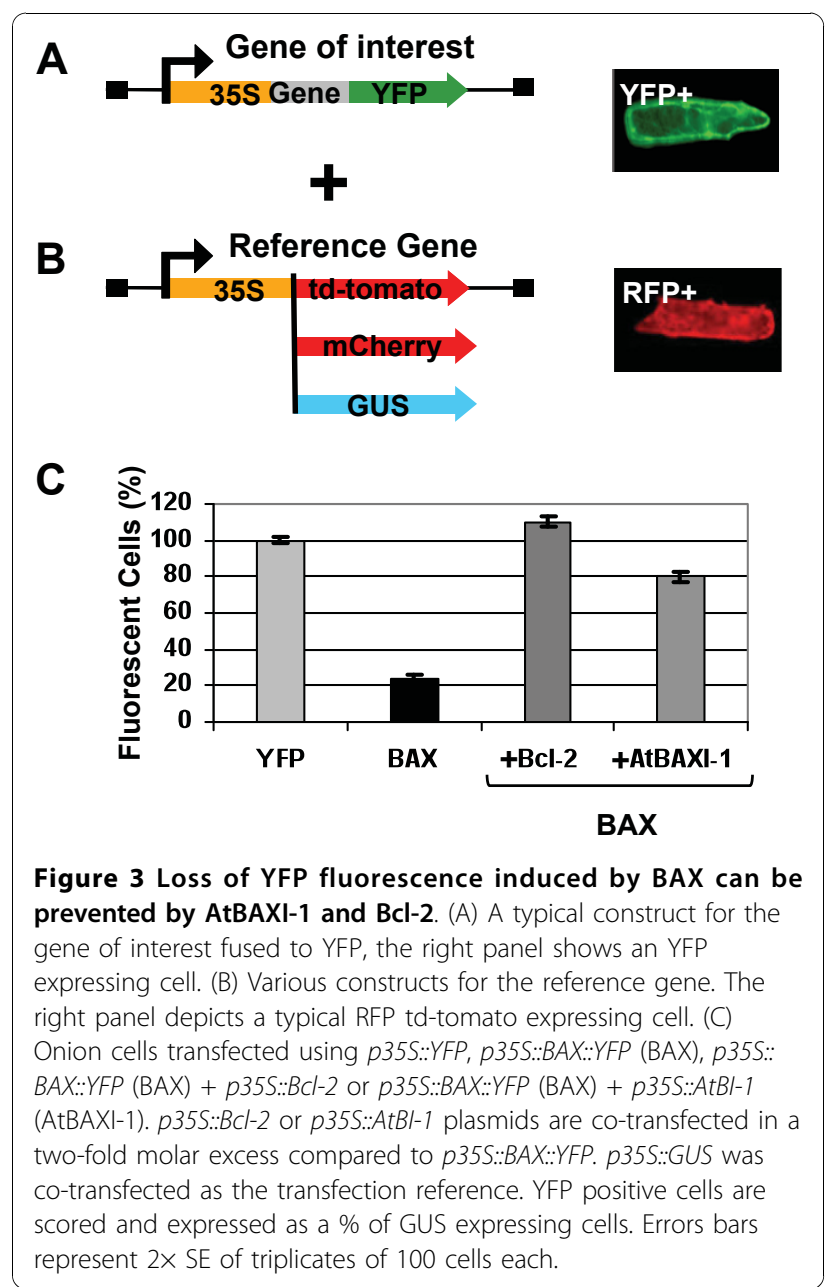


occurring during PCD. In this assay, every cell expresses both YFP and GUS. The number of YFP fluorescent cells is compared to the number of cells showing GUS enzymatic activity. $100 \%$ of GUS cells showing YFP fluorescence indicate no PCD. A percentage of YFP/ GUS cells below 100\% quantifies PCD in the population of transfected cells.

$48 \mathrm{~h}$ after bombardment, cells with YFP fluorescence were counted. After scoring, the cells were incubated with X-GLUC to count cells with GUS enzymatic activity. The result is given in Figure 3C. Bombarding YFP as a negative control gave a $100 \%$ of YFP fluorescent cells. When expressing the fusion BAX::YFP, only $20 \%$ of GUS positive cells were YFP positive. This implied that $80 \%$ of transfected cells had lost YFP fluorescence $48 \mathrm{~h}$ after bombardment. This value obtained with GUS as a reference gene, is the same as the value obtained in previous experiments using the RFP td-tomato gene as a reference (see Figure 2E). BAX::YFP and Bcl-2 co-transfection gave $100 \%$ of YFP positive cells, whereas $B A X::$ YFP and AtBI-1 co-transfection, gave $60 \%$ of YFP positive cells. The clear suppression effect of these two antiPCD genes on the loss of YFP fluorescence in BAX::YFP expressing cells further demonstrated that the loss of YFP signal can be taken as a measure of PCD.

\section{Use of YFP to visualise PCD in whole root tissue}

As a proof of principle that our method allows the study of PCD in whole tissues, we show here YFP fluorescence attenuation during xylem differentiation in Arabidopsis. As explained in the introduction, the end point of xylem differentiation is PCD. Events occurring during the PCD of plant tracheary elements developing in zinnia cell culture can be examine using various microscopic and labelling techniques [13], including caspase substrates [30]. There is, however, no report of such observations with xylem cells embedded in the root tissue, as it has not been possible. To detect in vivo a possible $\mathrm{pH}$ drop in the xylem cell of intact roots of Arabidopsis seedlings, an YFP::CesA7 fusion and a $m$ CherryER marker of the endoplasmic reticulum, both under the control of the cellulose synthase $I R X 3$ promoter were introduced into Arabidopsis Ler. The IRX3 promoter is xylem specific and is expressed when secondary cell wall deposition starts [31]. Studies using zinnia culture have shown that PCD occurs after cell wall deposition [32]. Therefore, xylem specific expression of YFP at the time of cell wall deposition is expected to facilitate the observation of the onset of PCD during xylem development in intact roots. $m$ CherryER, a $\mathrm{pH}$ insensitive RFP protein, has been introduced here to produce a stronger proof-of-principle experiment, but would not be required for routine scoring of cell death in tissues. Transgenic lines expressing both reporter genes were selected for a low/medium

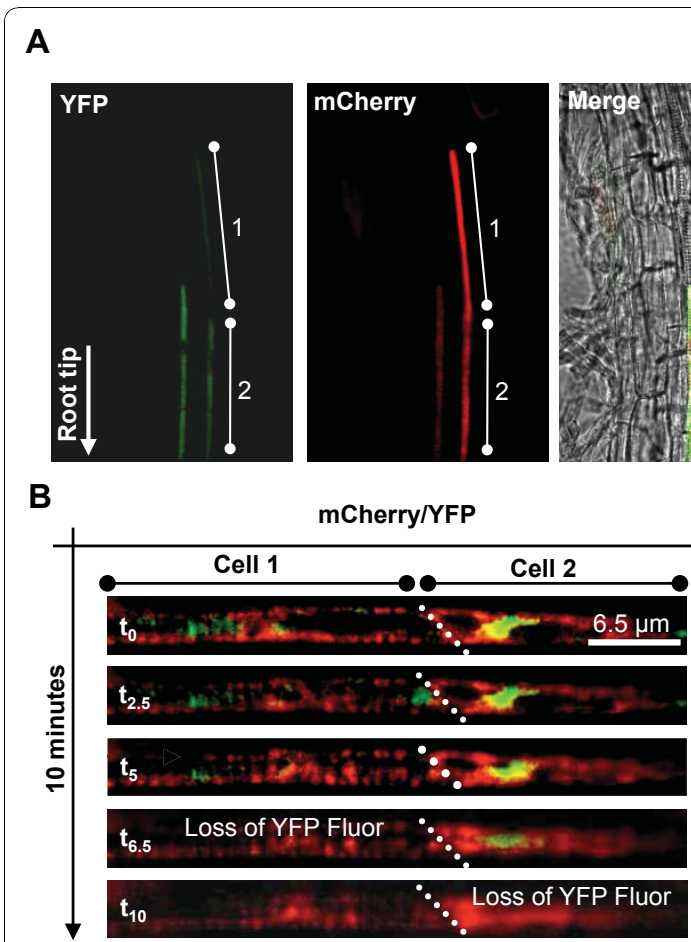

Figure 4 Loss of YFP fluorescence during xylem development. (A) Five day old Arabidopsis root expressing both YFP::CesA7 and mCherryER under the xylem specific promoter of the cellulose synthase subunit IRX3. White bars indicate two adjacent xylem cells (1 \& 2). Left panel shows YFP::CesA7 fluorescence. Centre panel shows mCherryER fluorescence. Right panel shows both the YFPCesA7 and mCherry ER fluorescence merged with the bright field image. ${ }^{*}$ highlights the typical banding pattern characteristic of xylem. Images were captured using a YFP/mCherry dual (51019) filter. (B) Time-lapse photography of two adjacent xylem cells (1 \& 2) in the same line as above. White dotted lines highlight the cell wall between cells 1 \& 2. Green: YFP fluorescence; red: mCherry fluorescence.

expression level with the fluorescent markers clearly observed deep within the root tissue. During the secondary-cell-wall deposition stage of xylem differentiation, both reporters were expressed and detected in the differentiating xylem only (Figure 4A). In Figure 4B, YFP and mCherryER are both seen expressed in two contiguous xylem cells. Previously, one of us (RW) has shown that the YFP::CesA7 protein fusion decorated transport vesicles in the cytoplasm, while mCherryER decorated the cortical ER [33]. mCherryER displayed a banding pattern typical of xylem cells during terminal differentiation (Figure 4A and 4B). As differentiation progressed away from the root tip, time-lapse photography of a file of two cells showed that in the cell distal to the root tip (Figure 4B, cell 1), YFP fluorescence was lost in the space of six minutes (Figure $4 \mathrm{~B}$ and Additional File 1, Movie S1), while mCherry fluorescence remained unaffected and this for 40 minutes (data not 
shown). The cell proximal to the root tip (cell 2) started to lose YFP fluorescence five minutes after cell 1 (Figure 4B and Additional file 1, Movie S1). For a given cell, the whole sequence lasted circa five to six minutes. It should be noted that six minutes represent the time taken by a single cell to lose YFP fluorescence and not the time taken for the cell to reach that point after PCD initiation. By comparison, the PCD process including time for BAX gene expression can take up to $48 \mathrm{~h}$ in Figure 2.

\section{Discussion}

We present here a method that uses YFP fluorescence as an indicator of the intracellular acidification that occurs during plant PCD. BAX expression in onion cells induced a loss of mitochondrial membrane potential, caspase-like activity and plasma membrane retraction, three hallmarks of plant PCD. We confirmed that the cytoplasmic $\mathrm{pH}$ drop from 7.3 to 5.5 described to occur during PCD in pollen tubes [4], occurred too in BAXinduced PCD in onion cells. We measured this $\mathrm{pH}$ change using the $\mathrm{pH}$ probe ptGFP, however this technique is too slow and labour intensive to score routinely PCD in plant cells. By contrast, we demonstrate here that the $\mathrm{pH}$ shift can be visualised much more simply as a loss of YFP fluorescence. The loss of YFP fluorescence correlated with induced caspase-like activity and could be inhibited by the PCD suppressors AtBI-1 and Bcl2. PCD can therefore be scored as absence of YFP fluorescence in cell expressing YFP using a fluorescence microscope and appropriate detection settings.

YFP can be expressed in the cell of choice either on its own or as a fusion to a protein of interest to confirm expression, providing the fusion does not affect protein function. The ability to measure the consequence of gene expression in a none-destructive manner is an important tool to study gene function in any particular process. The use of YFP fluorescence as a PCD marker means that data can be captured in real time including information on sub-cellular localisation and kinetics. Conveniently, RFP fluorescence is not affected by changes in intracellular $\mathrm{pH}$ and remains detectable until the very last stages of PCD. This makes RFP proteins convenient references for transfection or gene expression. For example, sub-cellular localisation or expression can be monitored after the $\mathrm{pH}$ shift by fusing the gene of interest to the RFP sequence. The results section above shows examples using RFP td-tomato, RFP mCherry and GUS as transfection reference. Any reporter protein found to exhibit the required stability at low$\mathrm{pH}$ would constitute a suitable alternative to these three proteins for use in our $\mathrm{pH}$ cell death assay.

As a demonstration of the usefulness of YFP as a PCD marker, we transiently expressed the mouse $B A X$ gene to induce PCD in onion epidermal cells. Animal $B A X$ has already been shown to induce PCD in plant cell [20]. Apoptosis induction in animal cells is linked with the localisation of BAX at the mitochondria, and the same BAX localisation is associated with PCD induction in plants [22]. $B A X$-induced PCD can be prevented by over expressing the plant PCD suppressor gene AtBI-1 $[20,34]$. We found $B A X$ to behave in the onion assay exactly as predicted from the published studies above, reinforcing the proposition that data obtained using $\mathrm{pH}$ shift as a marker of PCD correlate with the results obtained with other PCD markers. In addition, we found the animal anti-apoptotic gene $B c l 2$ to block $B A X$-induced cell death in plants, possibly through direct physical interaction between the two proteins as described in animal cells $[35,36]$. Incidentally, these experiments showed that AtBI-1 and human Bcl-2 both suppressed PCD upstream of the $\mathrm{pH}$ shift, providing some insight in the cell death cascade in plants. Finally, we show here that onion cells are suitable for PCD studies. These cells come as a single layer of flat cells, facilitating microscopic observation. Onion epidermal cells are easy to handle and transfect using biolistics. We found that in addition to a $\mathrm{pH}$ shift, other PCD markers can be detected in onion cells such as caspase-like activities, loss of mitochondrial membrane potential and plasma membrane retraction.

In addition to experiments carried out using transient expression, we show the application of our method to the observation of differentiating xylem cells in Arabidopsis roots. PCD in differentiating xylem can be readily studied in zinnia cell culture [32] but not inside intact tissue such as root, as xylem cells are surrounded by several layers of cells. Our preliminary experiment suggests that YFP could be used to visualise PCD during the normal development of xylem. To our knowledge this is a first report using a PCD marker in live root and further work is required to characterised PCD in that experimental system. Nevertheless, our experiment demonstrates the power of cell-specific expression of YFP to visualise in real time and in planta, the $\mathrm{pH}$ shift marker of PCD. This approach could be extended to other developmental cell death systems.

\section{Conclusion}

In conclusion, we show here that the fluorescence of cells expressing YFP is greatly reduced at a specific stage of PCD in plants. This observation is the basis of the PCD assay described here. Combined with gene coexpression systems, this assay provides a convenient tool to study both the genetic regulation and the cell biology of PCD. Additionally, because this loss of YFP fluorescence is a specific marker of $\mathrm{pH}$ during $\mathrm{PCD}$, it can be used to bring insights in the $\mathrm{pH}$ shift process itself. 
Finally, cell biologists may be unaware of this effect of PCD upon YFP fluorescence, which could lead to misinterpretation of expression data. To avoid this, a pH insensitive reporter should be selected.

\section{Methods}

\section{Plasmid DNA}

pART7 with CaMV 35S and Ptilosarcus GFP (PtGFP) was bought from Nanolight, pinetop, USA. Mouse $B A X$ alpha fused to the a yellow fluorescent protein (YFP) [37] and YFP (pEYFP-N1 \#6006-1 CLONTECH Laboratories, Inc.) clones were provided by A. Gilmore, Manchester UK, and cloned as Eco47-Xho1 fragments into pDH51 [38] cut with Sma1-Xho1. pDH51 provides a CaMV 35S promoter and terminator. A human Bcl2 cDNA clone was provided by T. Nishimoto, Kyushu Japan, and cloned as a BamH1-Sac1 fragment from pcDEB into pcGUS cut with BamH1-Sac1, providing a CaMV 35S promoter and NOS terminator. AtBAX-Inhibitor1 (At5g47120) was obtained from EST ATTS1836 via NASC, the European Arabidopsis Stock Centre, with the full coding sequence obtained by adding the sequence coding for MDAFSSF at the 5 ' end. The ORF was excised out of pBluescript SK+ into pDH51 using BamH1-Sal1. pRTL2-GUS expressing the beta-glucuronidase (GUS) gene is a gift from J. Carrington, College Station, USA. pAN57 containing mannosidase-tdtomato (RFP td-tomato) was obtained from Andreas Nebenführ [39]. All plasmid preps were performed using 'Nucleospin plasmid ' or 'Nucleobond Xtra midi plus' from Macherey-Nagel, Duren, BD.

\section{Bombardment Protocol}

Each onion slice was transfected with a total of $10 \mu \mathrm{g}$ of plasmid DNA. YFP and RFP plasmids were used in a 1:1 molar ratio. However, because GUS histochemical assays are more sensitive than YFP fluorescence detection, a dilution series of GUS plasmid concentration relative to YFP plasmid was carried out first to determine that a 1:1 ratio of cells expressing both GUS and YFP corresponded to a plasmid molar ratio of GUS 1:2.8 YFP. DNA was first precipitated onto $60 \mathrm{mg}$ aliquots of $1.6 \mu \mathrm{m}$ sterilised gold particles. Aliquots of gold particles were first vortexed for 30 seconds and then sonicated for 3 minutes. The DNA was then added in a volume under $80 \mu \mathrm{L}$, followed by $100 \mu \mathrm{L}$ of $2.5 \mathrm{M}$ $\mathrm{CaCl}_{2}$ and $20 \mu \mathrm{L}$ of spermine $0.1 \mathrm{M}$ with vortexing inbetween each addition. The mix was further vortexed for 3 minutes and then left on ice for 15 minutes. The supernatant was removed and the particles were washed in $500 \mu \mathrm{L}$ of ethanol. After 15 minutes on ice, the supernatant was removed and the particles were then washed again in ethanol this time using $200 \mu \mathrm{L}$. After one final 15-minute incubation on ice the supernatant was removed and the particles were re-suspended in 40 $\mu \mathrm{L}$ of ethanol. Bombardments were carried out on squares of onion fleshy scales of about $4 \mathrm{~cm}^{2}$ and $3 \mathrm{~mm}$ thick, each onion square was mounted on $1 \%$ agar plates to hold it in position to be fired upon and to prevent drying. A $10 \mu \mathrm{L}$ volume of particles was deposited onto the micro-projectile and left to dry before being used in the gun. Samples were fired using 1100 psi rupture discs in a PDS-1000/HE particle gun (BioRad) at a distance of $9 \mathrm{~cm}$ under a vacuum of -27 inches $\mathrm{Hg}$ (0.925 bar). Bombarded onion pieces were then kept epidermal side down on the agars plates, which were sealed with parafilm and kept at $23^{\circ} \mathrm{C}$ until use.

\section{YFP and RFP fluorescence}

YFP positive cells and RFP positive cells were scored using a Leica DM5500 fitted with a Photometrics cascade II 512B EMCCD camera (Photometrics UK) and a dual filter YFP/dsRED (part 51019; Chroma Technology Corp.).

\section{Mitotracker staining for mitochondria depolarisation}

Onion cells were incubated for five minutes in $100 \mathrm{nM}$ mitotracker red CMX ROS (Invitrogen) and observed immediately using a fluorescence microscope and a dual filter YFP/dsRED (part 51019; Chroma Technology Corp.).

\section{In situ caspase activity}

Activity was detected using the cell permeable and labelled Val-Ala-Asp peptide: FAM-VAD-FMK (APO LOGIX). Onion cells were incubated for 10 minutes in $4 \mu \mathrm{l}$ of the $30 \times$ working dilution of the inhibitor diluted in $300 \mu \mathrm{l}$ of water. Cells were washed twice in $5 \mathrm{ml}$ of sterile distilled water before microscopic observation.

\section{Fluorescence ratio imaging of ptGFP}

Fluorescence ratios were calculated in accordance to Schulte et al. (2006) [25]. Briefly, fluorescence images at excitation wavelengths of $475 \mathrm{~nm}$ and $390 \mathrm{~nm}$ were taken using a Nikon TE2000 PFS Widefield FRAP microscope fitted with a Cascade II EMCCD camera using a $10 \times / 0.30$ plan fluor objective. For this, cells expressing ptGFP were excited using a GFP filter (480/ $20)$ or a DAPI filter $(402 / 15)$ with the emission wavelength centred on $540 \mathrm{~nm}$. Images for each excitation were captured using Metamorph acquisition software from Molecular Devices. The ImageJ 1.42 free software was used to calculate a signal intensity value for every image. The ratio was subsequently calculated for the signal values obtained for an excitation at $475 \mathrm{~nm}$ and at $390 \mathrm{~nm}$. 


\section{Calibrating ptGFP signal}

A calibration curve was created by incubating ptGFPexpressing cells in buffers at $\mathrm{pH}$ ranging from 5 to 8 . For this, F475/F390 ratiometric readings were taken in epidermal cells expressing PtGFP and incubated in solutions buffered at a $\mathrm{pH}$ ranging from 5 to 8 . For this, 30 hours after bombardment, onion epidermal cell peels transfected with PtGFP were incubated in 6 wells plates, each well containing $3 \mathrm{~mL}$ of $\mathrm{pH}$-buffered solution and $0.003 \%$ Triton-X-100. Triton X-100 was added in order to permeabilise cell membranes and to facilitate equilibration with the extra cellular buffer. Buffers used were: sodium acetate $50 \mathrm{mM}, \mathrm{pH} 5$, MES (2-( $N$-morpholino) ethanesulfonic acid) $50 \mathrm{mM}, \mathrm{pH} 6$ and 7 , and Tris- $\mathrm{HCl}$ (Tris (hydroxymethyl) aminomethane hydrochloride) 50 $\mathrm{mM}, \mathrm{pH}$ 8. Onion epidermal peels were incubated in these solutions for 1 hour, with slow shaking before fluorescence analysis.

\section{GUS staining epidermal peels}

Tissue samples were submerged in GUS staining solution (sodium phosphate buffer at $\mathrm{pH}$ 7, $10 \mathrm{mM}$ EDTA, $0.1 \%$ Triton X-100, $2 \mathrm{mM}$ potassium ferricyanide and 2 $\mathrm{mM}$ potassium ferrocyanide). For every one $\mathrm{mL}$ of this staining solution used, $1 \mu \mathrm{l}$ of 5 -Bromo-4-chloro-3-indolyl $\beta$-D-glucuronide (X-Gluc) (Melford, UK) was added from a stock at $50 \mathrm{mg} / \mathrm{ml}$ in dimethyl sulfoxide (DMSO). Samples submerged in the stain were vacuum infiltrated for 3 minutes, followed by 16 hours incubation at $37^{\circ} \mathrm{C}$ in the dark (plates were sealed with parafilm and then wrapped in tin foil to exclude light). The GUS stain solution was then removed and the tissue samples fixed in $70 \%$ ethanol.

\section{Fluorescent microscopy of roots}

The constructs and the line expressing both the YFPCesA7 fusion (Cellulose synthase subunit A7) and the mCherry endoreticulum (ER) reporter have been described in Wightman et al. 2008 [40]. Seedlings were grown in continuous light on vertical $0.5 \times \mathrm{MS}$ salts (Duchefa), 1.5\% agar plates. Root images were captured on a DMR microscope using an YFP/mCherry dual (51019) filter, a HCX PL APO CS $\times 63$ water NA 1.2 objective and a SPOT Xplorer 4MP camera.

\section{Additional material}

Additional file 1: AVI Movie - PCD in xylem cells of Arabidopsis roots. Three adjacent root cells expressing YFP::CesA7 and mCherryER under control of the xylem-specific promoter from the cellulose synthase subunit IRX3. Arabidopsis seedlings were grown for 5 days in continuous light on vertical $0.5 \mathrm{MS}, 1.5 \%$ agar plates. Images were captured with a Leica DMR microscope using a YFP/mCherry dual (51019) filter, a HCX PL APO CS $\times 63$ water NA 1.2 objective and a SPOT Xplorer 4MP camera.

\section{Acknowledgements}

We thank C. Plieth for very helpful advice on the use of PtGFP and A. Day for kindly giving us access to his PDS-1000/HE particle gun. The Bioimaging Facility microscopes used in this study were purchased with grants from BBSRC, Wellcome Trust and the University of Manchester Strategic Fund. Special thanks go to Peter March and Robert Fernandez for their help with microscopy. BY holds a DTA BBSRC studentship.

\section{Author details}

${ }^{1}$ Faculty of Life Sciences, Michael Smith Building, University of Manchester, Oxford Road, Manchester M13 9PT, UK. Biochimie et Physiologie Moléculaire des Plantes, CNRS UMR 5004, 2 place Viala, 34060 Montpellier Cedex 1, France.

\section{Authors' contributions}

BY participated to the conception the study, made constructs, carried out $\mathrm{pH}$ measurements, transient assays and drafted the manuscript. RW made constructs, transgenic lines and took xylem movies. RB conceived part of the study and carried out preliminary experiments, SP participated in $\mathrm{pH}$ measurements. PG conceived the study and participated in its design and coordination and drafted the manuscript. All authors read and approved the final manuscript.

\section{Competing interests}

The authors declare that they have no competing interests.

Received: 3 August 2010 Accepted: 30 November 2010 Published: 30 November 2010

\section{References}

1. O'Brien IEW, Reutelingsperger CPM, Holdaway KM: Annexin-V and TUNEL use in monitoring the progression of apoptosis in plants. Cytometry 1997, 29:28-33.

2. Bonneau L, Ge Y, Drury GE, Gallois P: What happened to plant caspases? Exp Bot 2008, 59:491-499.

3. Bedner E, Smolewski P, Amstad P, Darzynkiewicz Z: Activation of caspases measured in situ by binding of fluorochrome-labeled inhibitors of caspases (FLICA): correlation with DNA fragmentation. Exp Cell Res 2000, 259:308-313.

4. Bosch M, Franklin-Tong VE: Temporal and spatial activation of caspaselike enzymes induced by self-incompatibility in Papaver pollen. Proc Natl Acad Sci USA 2007, 104:18327-18332.

5. Zhang L, Xu Q, Xing D, Gao C, Xiong H: Real-Time Detection of Caspase3-Like Protease Activation in Vivo Using Fluorescence Resonance Energy Transfer during Plant Programmed Cell Death Induced by Ultraviolet C Overexposure. Plant Physiol 2009, 150:1773-1783.

6. Vacca RA, de Pinto MC, Valenti D, Passarella S, Marra E, De Gara L: Production of Reactive Oxygen Species, Alteration of Cytosolic Ascorbate Peroxidase, and Impairment of Mitochondrial Metabolism Are Early Events in Heat Shock-Induced Programmed Cell Death in Tobacco Bright-Yellow 2 Cells. Plant Physiol 2004, 134:1100-1112.

7. Yu X, Perdue T, Heimer $Y$, Jones A: Mitochondrial involvement in tracheary element programmed cell death. Cell Death Differ 2002, 9:189-198.

8. Kerry G, Mark W: The use of chloromethyl-X-rosamine (Mitotracker Red) to measure loss of mitochondrial membrane potential in apoptotic cells is incompatible with cell fixation. Cytometry 1999, 36:355-358.

9. Widholm JM: The Use of Fluorescein Diacetate and Phenosafranine for Determining Viability of Cultured Plant Cells. Biotech \& Histochem 1972, 47:189-194.

10. Truernit $E$, Haseloff J: A simple way to identify non-viable cells within living plant tissue using confocal microscopy. Plant Meth 2008, 4:15.

11. Jacyn Baker C, Mock NM: An improved method for monitoring cell death in cell suspension and leaf disc assays using Evans blue. Plant Cell Tiss Org Cult 1994, 39:7-12.

12. Swanson SJ, Bethke PC, Jones RL: Barley Aleurone Cells Contain Two Types of Vacuoles: Characterization of Lytic Organelles by Use of Fluorescent Probes. Plant Cell 1998, 10:685-698. 
13. Groover A, DeWitt N, Heidel A, Jones A: Programmed cell death of plant tracheary elements differentiating in vitro. Protoplasma 1997, 196:197-211.

14. Obara K, Kuriyama $\mathrm{H}$, Fukuda $\mathrm{H}$ : Direct evidence of active and rapid nuclear degradation triggered by vacuole rupture during programmed cell death in Zinnia. Plant Physiol 2001, 125:615-626.

15. Jones AM: Programmed Cell Death in Development and Defense. Plant Physiol 2001, 125:94-97.

16. Rotari $V$, He R, Gallois P: Death by proteases in plants: whodunit. Physiol Plant 2005, 123:376-385.

17. Zhao J, Connorton JM, Guo Y, Li X, Shigaki T, Hirschi KD, Pittman JK Functional studies of split Arabidopsis $\mathrm{Ca} 2+/ \mathrm{H}+$ exchangers. J Biol Chem 2009:34075-34083.

18. Klein TM, Wolf ED, Wu R, Sanford J: High velocity microprojectiles for delivering nucleic acids into living cells. Nature 1987, 327:70-73.

19. Wrzaczek M, Brosché M, Kollist H, Kangasjärvi J: Arabidopsis GRI is involved in the regulation of cell death induced by extracellular ROS. Proc Natl Acad Sci USA 2009, 106:5412-5417.

20. Lacomme C, Santa Cruz S: Bax-induced cell death in tobacco is similar to the hypersensitive response. Proc Natl Acad Sci USA 1999, 96:7956-7961.

21. Kawai-Yamada M, Ohori $Y$, Uchimiya H: Dissection of Arabidopsis Bax Inhibitor-1 Suppressing Bax-, Hydrogen Peroxide-, and Salicylic AcidInduced Cell Death. Plant Cell 2004, 16(1):21-32.

22. Yoshinaga K, Arimura S, Hirata A, Niwa Y, Yun D, Tsutsumi N, Uchimiya H, Kawai-Yamada M: Mammalian Bax initiates plant cell death through organelle destruction. Plant Cell Rep 2005:408-417.

23. Saviani EE, Orsi CH, Oliveira JF, Pinto-Maglio CA, Salgado I: Participation of the mitochondrial permeability transition pore in nitric oxide-induced plant cell death. FEBS Lett 2002, 510:136-140.

24. Reape TJ, Molony EM, McCabe PF: Programmed cell death in plants: distinguishing between different modes. J Exp Bot 2008, 59:435-444.

25. Schulte A, Lorenzen I, Böttcher M, Plieth C: A novel fluorescent pH probe for expression in plants. Plant Meth 2006, 2(2):7.

26. Kneen M, Farinas J, Li Y, Verkman A: Green fluorescent protein as a noninvasive intracellular pH indicator. Biophys J 1998, 74:1591.

27. Llopis J, McCaffery J, Miyawaki A, Farquhar M, Tsien R: Measurement of cytosolic, mitochondrial, and Golgi pH in single living cells with green fluorescent proteins. Proc Natl Acad Sci USA 1998, 95:6803-6808.

28. Kimura S, Noda T, Yoshimori T: Dissection of the autophagosome maturation process by a novel reporter protein, tandem fluorescenttagged LC3. Autophagy 2007, 3:452-460.

29. Mitsuhara I, Malik KA, Miura M, Ohashi Y: Animal cell-death suppressors Bcl-xL and Ced-9 inhibit cell death in tobacco plants. Curr Biol 1999, 9:775-778, S771.

30. Twumasi P, lakimova ET, Qian T, van leperen W, Schel JH, Emons AM, van Kooten $\mathrm{O}$, Woltering EJ: Caspase inhibitors affect the kinetics and dimensions of tracheary elements in xylogenic Zinnia (Zinnia elegans) cell cultures. BMC Plant Biol 10:162

31. Gardiner JC, Taylor NG, Turner SR: Control of cellulose synthase complex localization in developing xylem. Plant Cell 2003, 15:1740-1748.

32. Fukuda H: Programmed cell death of tracheary elements as a paradigm in plants. Plant Mol Biol 2000, 44:245-253.

33. Wightman R, Marshall R, Turner SR: A Cellulose Synthase-Containing Compartment Moves Rapidly Beneath Sites of Secondary Wall Synthesis. Plant Cell Physiol 2009, 50:584-594.

34. Ihara-Ohori Y, Nagano M, Muto S, Uchimiya H, Kawai-Yamada M: Cell Death Suppressor Arabidopsis Bax Inhibitor-1 is Associated with Calmodulin Binding and Ion Homeostasis. Plant Physiol 2007:650-660.

35. Mahajan NP, Linder K, Berry G, Gordon G, Heim R, Herman B: Bcl-2 and Bax interactions in mitochondria probed with green fluorescent protein and fluorescence resonance energy transfer. Nat Biotechnol 1998, 16:547-552.

36. Hossini $\mathrm{AM}$, Eberle J: Apoptosis induction by $\mathrm{BCl}-2$ proteins independent of the BH3 domain. Biochem Pharm 2008, 76:1612-1619.

37. Valentijn AJ, Metcalfe AD, Kott J, Streuli CH, Gilmore AP: Spatial and temporal changes in Bax subcellular localization during anoikis. J Cell Biol 2003, 162:599-612.

38. Pietrzak M, Shillito R, Hohn T, Potrykus I: Expression in plants of two bacterial antibiotic resistance genes after protoplast transformation with a new plant expression vector. Nucleic Acids Res 1986, 14:5857-5868.

39. Nebenführ A, Gallagher L, Dunahay T, Frohlick J, Mazurkiewicz A, Meehl J, Staehelin L: Stop- and- Go Movements of the plant Golgi Stacks Are Mediated by the Acto-Myosin System. Plant Physiol 1999, 121:1127-1141.
40. Wightman R, Turner SR: The roles of the cytoskeleton during cellulose deposition at the secondary cell wall. Plant J 2008, 54:794-805.

doi:10.1186/1746-4811-6-27

Cite this article as: Young et al:: $\mathrm{pH}$-sensitivity of YFP provides an intracellular indicator of programmed cell death. Plant Methods 2010 $6: 27$.

\section{Submit your next manuscript to BioMed Central and take full advantage of:}

- Convenient online submission

- Thorough peer review

- No space constraints or color figure charges

- Immediate publication on acceptance

- Inclusion in PubMed, CAS, Scopus and Google Scholar

- Research which is freely available for redistribution
Ciomed Central 